\title{
Inducing a Curl with a Stretch
}

\section{Patterning grooves into the surface of an elastic ribbon can cause the ribbon to curl into a tube shape when it is stretched.}

\section{By Katherine Wright}

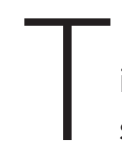
o make materials that will bend or curl on demand in a predefined way, researchers have traditionally sandwiched together two or more substances with different thermal expansion coefficients. When heated, the substances expand by different amounts, leading to a mismatch between their lengths that induces a curvature. Now, Emmanuel Siéfert of the Free University of Brussels, Belgium, and colleagues have found a way to induce bending by stretching a single substance [1]. Siéfert says that the discovery came by chance: He and his colleagues had been making so-called baromorphs-thin, balloon-like structures that can be inflated into preprogrammed shapes-when they observed the behavior.

In their experiments, the team used a 3D-printed mold to pattern a rubber ribbon with a series of parallel grooves. The grooves ran across the width of the roughly 2-cm-wide ribbon and had a spacing of around $0.5 \mathrm{~mm}$. Pulling on the ends of the ribbon, they observed that the ribbon curled into a tube with a consistent radius.

Changing the angle that the grooves were oriented relative to

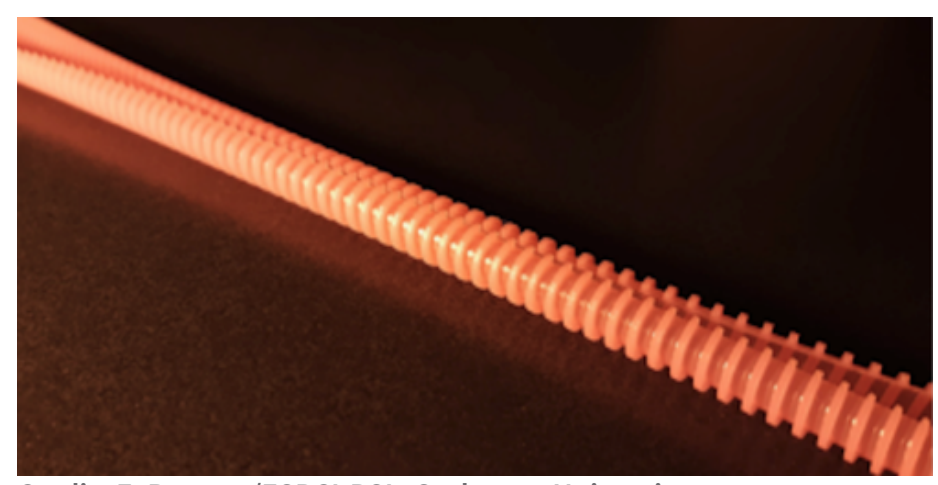

Credit: E. Reyssat/ESPCI-PSL, Sorbonne University

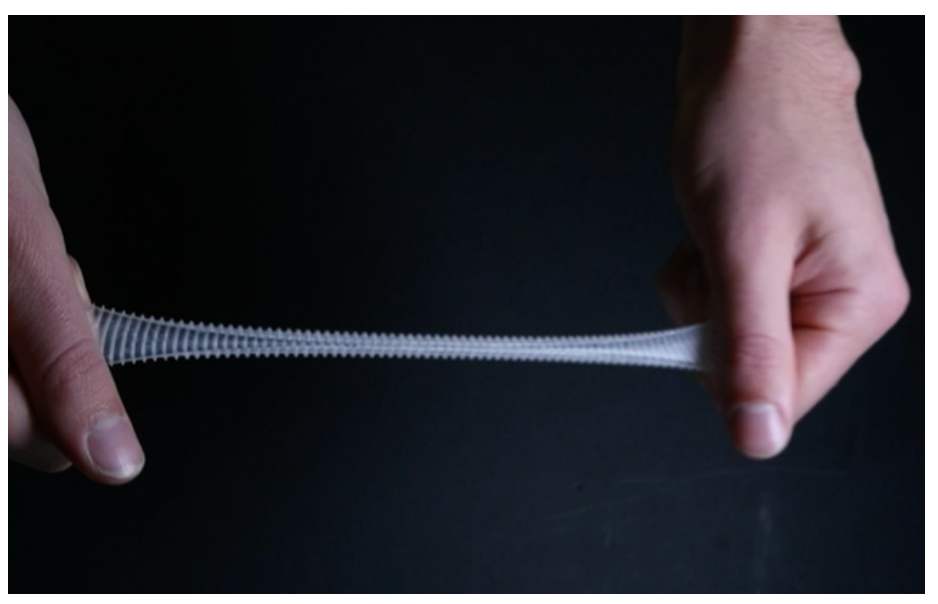

Video 1: When stretched, the edges of a ribbed ribbon curl inwards, transforming the flat system into a tube.

Credit: E. Siéfert et al. [1]

the width of the ribbon, the team found that they could control the tube formation process. They created tubes with the grooves on the outside $\left(0^{\circ}\right)$ and on the inside $\left(45^{\circ}\right)$, and they also suppressed tube formation completely $\left(30^{\circ}\right)$.

Siéfert says that he sees the ribbons being used to create small grippers (with the grooves on the outside) for tiny handles. And he thinks other potential applications will likely arise as the community working on mechanical metamaterials explores these materials further.

Katherine Wright is the Deputy Editor of Physics.

\section{REFERENCES}

1. E. Siéfert et al., "Stretch-induced bending of soft ribbed strips," Phys. Rev. Lett. 127, 168002 (2021). 


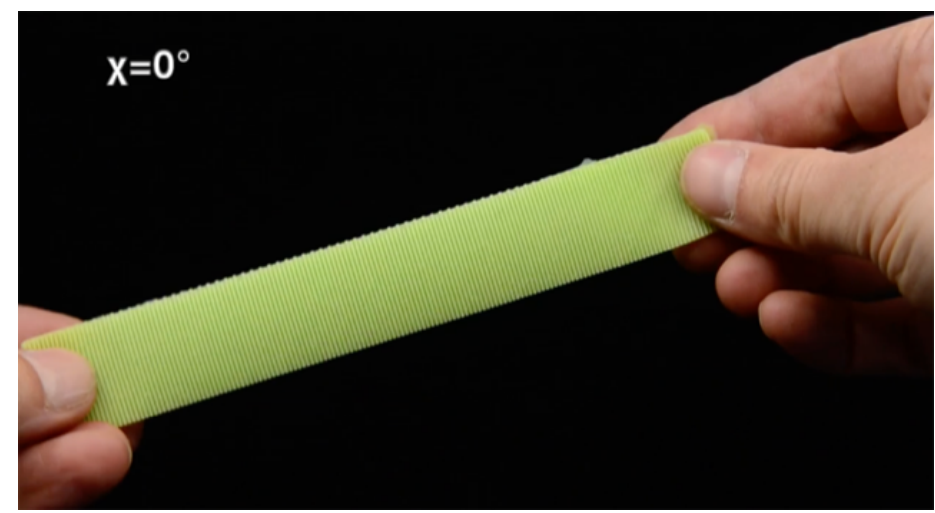

Video 2: The angle of the grooves relative to the width of the ribbon determines how the ribbon curls when stretched. Credit: E. Siéfert et al. [1] 